\title{
Transient Inactivation of the Neonatal Ventral Hippocampus Permanently Disrupts the Mesolimbic Regulation of Prefrontal Cholinergic Transmission: Implications for Schizophrenia
}

\author{
Julie M Brooks', Martin Sarter ${ }^{2}$ and John P Bruno*,' \\ 'Department of Psychology, The Ohio State University, Columbus, OH, USA; ${ }^{2}$ Department of Psychology, University of Michigan, \\ Ann Arbor, MI, USA
}

\begin{abstract}
These experiments determined the mesolimbic modulation of cortical cholinergic transmission in a neurodevelopmental model of schizophrenia. Mesolimbic-cholinergic abnormalities are hypothesized to contribute to the cognitive deficits seen in schizophrenia. Stimulation of NMDA receptors in nucleus accumbens (NAC) increases acetylcholine (ACh) release in the prefrontal cortex (PFC), a mechanism recently demonstrated to contribute to the control of attentional performance. We determined the ability of intra-NAC administration of NMDA to increase prefrontal ACh levels in adult rats that had received bilateral infusions of tetrodotoxin (TTX) to transiently interrupt impulse flow in the ventral hippocampus $(\mathrm{VH})$ during development. Rats received infusions of TTX or saline on postnatal day 7 (PD7) or day 32 (PD32), and the effects of NAC NMDA receptor stimulation on prefrontal cholinergic neurotransmission were assessed in adulthood. In animals treated as controls on PD7, NMDA increased prefrontal ACh levels by $121 \%$ above baseline. In contrast, PD7 infusions of TTX into the $\mathrm{VH}$ abolished the ability of NAC NMDA to activate prefrontal cholinergic neurotransmission (7\% increase). In animals that received TTX infusions on PD32, NMDA-evoked cholinergic activity did not differ from controls, indicating a restricted, neonatal critical period during which $\mathrm{VH} T \mathrm{TX}$ impacts the organization of mesolimbic-basal forebrain-cortical circuitry. Importantly, the failure of NAC NMDA to evoke cholinergic activity in rats treated with TTX on PD7 did not reflect a reduced excitability of corticopetal cholinergic neurons because administration of amphetamine produced similar elevations of prefrontal ACh levels in PD7 TTX and PD7 control animals. A third series of experiments demonstrated that the effects of PD7 TTX are a specific consequence of transient disruption of impulse flow in the $\mathrm{VH}$. Intra-NAC NMDA evoked prefrontal ACh release in rats receiving TTX, on PD7, into the dorsal hippocampus $(\mathrm{DH})$, basolateral amygdala, or NAC. Thus, impulse flow specifically within the $\mathrm{VH}$, during a sensitive period of development, is necessary for the functional organization of a mesolimbic-cortical circuit known to mediate attentional control processes. Therefore, neonatal inactivation of $\mathrm{VH}$ represents an effective animal model for studying the basis of certain cognitive symptoms of schizophrenia.
\end{abstract}

Neuropsychopharmacology (20II) 36, 2477-2487; doi: I0.1038/npp.20I I.I36; published online 3 August 201 I

Keywords: schizophrenia; acetylcholine; hippocampus; prefrontal cortex; nucleus accumbens; cognition

\section{INTRODUCTION}

Abnormalities in functional connectivity between cortical and subcortical brain regions are thought to represent a critical pathophysiological signature in schizophrenia (SZ; Jones, 2010). Dysregulated interactions within a broad distributed neural system involving the hippocampus (Lisman et al, 2010), prefrontal cortex (PFC; Wolf et al, 2009), ventral striatum (nucleus accumbens (NAC); Guillin et al, 2007), basal forebrain (Hyde and Crook, 2001), and

*Correspondence: Dr JP Bruno, Department of Psychology, The Ohio State University, Columbus, OH 43210, USA, Tel: + I 614 292 1770, Fax: + I 614688 4733, E-mail: bruno. @@osu.edu

Received 3 April 2011; revised 12 June 2011; accepted 22 June 201। midline thalamic nuclei (Welsh et al, 2010) have been linked to many of the cognitive symptoms seen in SZ, including deficits in attention, working memory, and cognitive flexibility.

There is also considerable evidence indicating that SZ reflects a complex set of neurodevelopmental disruptions and the ensuing dysregulations within the neural systems cited above lead to a reverberating maturation of anomalous circuits. This etiological perspective is currently being investigated using a wide range of developmental models in animals, including genetic risk factors (Lisman et al, 2008), perinatal infection (Patterson, 2009), early exposure to NMDA antagonists (Mouri et al, 2007), and early disruption of component regions, such as the ventral hippocampus (VH) (Tseng et al, 2009). 
Perhaps, the most widely studied of these animal models involves excitotoxic damage to the $\mathrm{VH}$ during neonatal development (for a review see, Tseng et al, 2009). Rats sustaining lesions of the $\mathrm{VH}$ as neonates exhibit a wide range of neuropathologies believed to contribute to the cognitive symptoms of SZ, including functional deficits in hippocampal (Francois et al, 2009) and prefrontal interneurons (Mitchell et al, 2005; Francois et al, 2009), abnormal dopamine-glutamate regulation of prefrontal and accumbens neuronal excitability (Tseng et al, 2007), reduced dendritic spine density in PFC (Marquis et al, 2008), and dysregulated cortical acetylcholine (ACh) release (Alexander et al, 2009; Laplante et al, 2004). Not surprisingly, animals with such lesions also develop abnormal behaviors during or after adolescence that resemble those seen in SZ, including hypersensitivity to stimulants and NMDA antagonists (Flores et al, 1996; Lipska et al, 2002b), impairments in working memory (Brady et al, 2010; Lipska et al, 2002a), and deficits in cognitive flexibility (Marquis et al, 2008). The interpretation for these enduring neurobehavioral effects of $\mathrm{VH}$ damage has been that loss of activity, during sensitive periods, within intrinsic hippocampal neurons as well as hippocampal projection neurons to critical targets such as PFC and NAC permanently alters the functional maturation of neuronal targets within the distributed system.

Despite the valuable evidence derived from research on animals with lesions of the neonatal $\mathrm{VH}$, it is burdened by minimal etiological validity due to the extensive cell loss produced by the lesion. In the human condition, evidence indicating hippocampal pathology in SZ suggests much more subtle alterations, with a disarray of the typical orientation of pyramidal neurons and a decline in the number of synapses (Goldman and Mitchell, 2004; Harrison, 2004). These effects have been speculated to contribute to the emergence of an imprecise (ie, poor signal-to-noise ratios) and mis-timed information flow rather than the complete absence of neuronal output (Ford et al, 2007; Tamminga et al, 2010). In an effort to enhance the validity of this model, we and others have studied animals after transiently interfering with impulse flow in the developing $\mathrm{VH}$, caused by local infusions of tetrodotoxin (TTX), a potent and selective blocker of voltage-sensitive $\mathrm{Na}^{+}$channels (Narahashi, 1972). Several studies indicated that such a disruption of $\mathrm{VH}$ impulse flow during a critical developmental period affects the functional maturation of VH efferent circuitry not unlike those observed following lesions of the VH. Specifically, Lipska et al (2002b) demonstrated that intra-VH TTX on postnatal day 7 (PD7) resembled those with permanent neonatal hippocampal lesions in their exaggerated sensitivity to the motoric effects of amphetamine and NMDA antagonists. More recently, Louillot and co-workers (Meyer et al, 2009; Peterschmitt et al, 2007) reported that intra-VH infusions of TTX to rat pups on PD8 produced deficits in latent inhibition and associated changes in accumben's DA release when tested as adults.

We extended our research on the neurochemical disruptions of intra- $\mathrm{VH}$ infusions of TTX during early development (PD7) by determining the effects of the transient inactivation on the accumben's (NAC) regulation of the basal forebrain cortical cholinergic system (BFCS).
We previously reported that, in intact rats, intra-NAC administration of NMDA stimulates the release of ACh in prefrontal, but not in parietal cortex (Zmarowski et al, 2005, 2007). Intra-NAC NMDA administration facilitates attentional performance under conditions of enhanced demands on performance, suggesting that NAC-cholinergic interactions mediate the motivated recruitment of attention systems (St Peters et al, 2011). This evidence is consistent with the hypothesis that stimulation of NAC NMDA receptors mimics the top-down PFC-NAC activation of the BFCS, an activation that is critical for the recruitment of motivation to stay on task when faced with enhanced cognitive load. Deficits in such top-down regulation of attentional processes have been demonstrated in SZ patients (Nuechterlein et al, 2009). In this study, we determined whether a transient inactivation of neuronal activity within $\mathrm{VH}$ at the same developmental stage would produce similarly enduring limbic-cortical deficits. In addition, we also examined the age-dependency (ie, critical period) of this effect by determining the effects of intra-hippocampal administration of TTX at postnatal day 32 (PD32). Finally, the anatomical specificity of this effect was addressed by measuring the effect of TTX-induced inactivation on PD7 in dorsal hippocampus (DH) as well as two critical target regions of the $\mathrm{VH}$, the $\mathrm{NAC}$ and the basal lateral amygdala (BLA) at PD7.

\section{MATERIALS AND METHODS}

\section{Animals}

Male Wistar rats (Charles River, Wilmington, MA) were used. Neonatal rats were born in our breeding colony and were weaned on PD21. Post-weaning, animals were housed in groups of 2-3 rats in plastic cages lined with corn cob bedding (Harlan Teklad, Madison, WI) and had access to food and water ad libitum. On PD56, rats were individually housed to accommodate/protect microdialysis guide cannula head-stages. Animals were maintained on a $12: 12 \mathrm{~h}$ light/dark cycle (lights on $0600 \mathrm{~h}$ ) in a temperature- and humidity-controlled AAALAC-approved animal facility. All procedures were approved by The Ohio State University Institutional Animal Care and Use Committee in accordance with the NIH Guide for the Care and Use of Laboratory Animals.

\section{Surgical Procedures}

Transient inactivation of developing hippocampus. Neonatal ventral hippocampal inactivation: Neonatal surgical procedures were performed in pups on PD7 (body weight: $15-18 \mathrm{~g}$ ). Male pups were randomly assigned to receive a sham (nVHSham) or TTX (nVHTTX) injection and anesthetized by hypothermia (placed on ice for 8-10 min). Pups were secured with tape onto a styrofoam platform that was positioned in a stereotaxic frame. An incision was made in the skin and the skull was penetrated with an infusion syringe (10 $\mu$ l syringe, $26 \mathrm{G}$ needle) at coordinates: AP, $-3.0 \mathrm{~mm}$; ML, $\pm 3.5 \mathrm{~mm}$; and DV, $-5.0 \mathrm{~mm}$ relative to Bregma. TTX ( $100 \mu \mathrm{M}$; Sigma, St Louis, $\mathrm{MO}$ ) was then infused into the $\mathrm{VH}$ in a total volume of $0.3 \mu \mathrm{l}$ over a 2-min period. Following completion of the infusion, 
the needle was left in place for an additional 3 min to allow diffusion of TTX and prevent backflow up the needle tract. This infusion procedure was then repeated in the contralateral hemisphere. For sham surgery (nVHSham), $0.3 \mu \mathrm{l}$ of saline $(0.9 \%)$ was infused at the same rate. Saline and TTX infusions were tinted with Evans Blue dye $(2.5 \mathrm{mg}$ dye/1 ml saline vehicle; Sigma) to identify the site of injection during histological preparations. After surgery, the pups were warmed on a heating pad and returned as a group to the litter's nest area. Pups and dams were left undisturbed until weaning on PD21.

Neonatal dorsal hippocampal inactivation: In these pups (PD7), the DH was infused with TTX (nDHTTX) or a saline control (nDHSham). Surgical procedures and infusion parameters were the same as those described for nVHTTX pups, except that the following coordinates were used: AP, $-3.0 \mathrm{~mm}$; ML, \pm 2.6 ; and DV, $-3.2 \mathrm{~mm}$ relative to Bregma.

Periadolescent ventral hippocampal inactivation: Male rats, at $\mathrm{PD} 32$, were anesthetized with inhalant isofluorane $\left(2 \%, 0.61 / \mathrm{min}, \mathrm{O}_{2}\right)$. TTX $(100 \mu \mathrm{M}, 0.3 \mu \mathrm{l}$; paVHTTX) or saline $(0.9 \%$; paVHSham) was bilaterally infused into the $\mathrm{VH}$ (AP, $+3.2 \mathrm{~mm}$ from intra-aural line; $\mathrm{L}, \pm 4.4 \mathrm{~mm}$; and DV,$-7.2 \mathrm{~mm}$ ) at a rate of $0.15 \mu \mathrm{l} / \mathrm{min}$ with a syringe pump. The needle was left in place for $3 \mathrm{~min}$ and then withdrawn. The incision was closed using silk sutures and the area was swabbed with a topical antibiotic ointment (Neosporin). Following surgery, the animals were returned to their home cages and left undisturbed for 28 days.

Transient inactivation of regions within limbic-prefrontal circuit. Neonatal NAC inactivation: In these pups, the shell region of the NAC was infused with TTX (nNACTTX) or a saline control (nNACSham). Surgical procedures and infusion parameters were the same as those described for nVHTTX pups, with the following coordinates: AP, +6.5 (relative to intra-aural line); ML, \pm 1.3 ; and DV, $-5.9 \mathrm{~mm}$ relative to Bregma.

Neonatal basolateral amygdala inactivation: In these pups, the BLA was infused with TTX (nBLATTX) or a control volume of saline (nBLASham). Surgical procedures and infusion parameters were the same as those described for nVHTTX pups, with injections performed at a $4^{\circ}$ rostral angle at the following coordinates: $\mathrm{AP},-1.0 ; \mathrm{ML}, \pm 3.8$; and DV,$-6.0 \mathrm{~mm}$ relative to Bregma.

\section{Guide Cannula Implantation}

All animals were implanted with microdialysis guide cannula between PD56 and 70 under isoflurane anesthesia. Rats were implanted unilaterally (hemispheres counterbalanced within treatment groups) with guide cannulae (SciPro, Sanborn, NY) in the mPFC and the shell region of the NAC. The mPFC guide cannula $(0.38 \mathrm{~mm}$ o.d., $15 \mathrm{~mm}$ shaft) was implanted at a $20^{\circ}$ rostral angle at: AP, +4.2; ML, \pm 0.6 ; and $\mathrm{DV},-0.6 \mathrm{~mm}$ relative to Bregma. The NAC cannula $(0.38 \mathrm{~mm}$ o.d., $20 \mathrm{~mm}$ shaft $)$ was implanted at a $0^{\circ}$ (ie, vertical) angle at: $\mathrm{AP},+1.5 ; \mathrm{ML}, \pm 0.8$; and $\mathrm{DV}$, $-5.6 \mathrm{~mm}$ relative to Bregma. Cannulae were fixed to the skull using skull screws and dental cement. To prevent clogging of the cannulae shafts, stainless-steel stylets ending flush with the guide cannulae were inserted. Antibiotic ointment (Neosporin) was applied to the area around the incision, and the animals were returned to their home cages.

\section{General Microdialysis Procedures}

Animals were handled for 1 week before surgery, during which time they habituated (4-6h per day) to their microdialysis testing environment of clear plastic concentric dialysis bowls $(35 \mathrm{~cm}$ high $\times 38 \mathrm{~cm}$ deep: Carnegie Medicine, Stockholm, Sweden) lined with corn-cob bedding (Harlan Teklad, Madison, WI). On the fourth day following surgery, animals were placed in their testing environment. Following a $30 \mathrm{~min}$ acclimation period, animals' dummy stylets were removed and microdialysis probes (SciPro, $0.2 \mathrm{~mm}$ o.d., $3.0 \mathrm{~mm}$ membrane tip for mPFC, $2.0 \mathrm{~mm}$ membrane tip for NAC) were inserted into the appropriate guide cannulae. Probes were continuously perfused $(1.25 \mu \mathrm{l} / \mathrm{min})$ with artificial cerebral spinal fluid (aCSF; containing in mM: $\mathrm{NaCl} 126.5, \mathrm{NaHCO}_{3} 27.5, \mathrm{KCl}$ 2.4, $\mathrm{CaCl}_{2} 1.2, \mathrm{Na}_{2} \mathrm{SO}_{4} 0.5, \mathrm{KH}_{2} \mathrm{PO}_{4} 0.5$, glucose 1.0, $\mathrm{pH}$ 7.1) during the microdialysis session. Dialysates were collected every $15 \mathrm{~min}$ during experimental sequences that were conducted using the following format. After the four baseline collections, the syringe was switched from aCSF to one containing NMDA $(150 \mu \mathrm{M})$. Following a 15-min washout, four additional collections were taken. The syringe was then switched back to one containing aCSF. Following a 15-min washout, three additional collections were taken. Immediately following session completion, probes were removed from the cannulae and stylets were replaced in the cannulae. Animals were then returned to their home cages. Between dialysis sessions, each dialysis probe was stored in an antibacterial agent (ProClin 150 Reagent; BAS, West Lafayette, IN) to ensure sterility. In all experiments, microdialysis sessions followed a repeated perfusion paradigm, with animals receiving at least one day between two successive microdialysis sessions.

\section{ACh Quantification}

ACh samples were stored $\left(-80^{\circ} \mathrm{C}\right)$ until analyzed using high-performance liquid chromatography with electrochemical detection. Using an autosampler (ESA, Chelmsford, MA), $15 \mu \mathrm{l}$ of each sample was injected onto a UniJet microbore analytical column $\left(1 \times 50 \mathrm{~mm}^{2}\right.$, BAS $)$ using a sodium phosphate mobile phase $\left(35 \mathrm{mM} \mathrm{Na}_{2} \mathrm{HPO}_{4}\right.$, $483.55 \mu \mathrm{M}$ EDTA, $0.005 \%$ microbicide reagent ProClin, $\mathrm{pH}=8.5$; flow rate of $0.15 \mathrm{ml} / \mathrm{min}$ ) to separate $\mathrm{ACh}$ and choline. Following separation of $\mathrm{ACh}$, a post-column enzyme reactor (BAS) was used to convert $\mathrm{ACh}$ into the reporting molecule $\mathrm{H}_{2} \mathrm{O}_{2} \cdot \mathrm{H}_{2} \mathrm{O}_{2}$ was quantified with a peroxidase-wired glassy carbon electrode (Model No. 5041 microdialysis analytical cell; ESA) at an applied potential of $-200 \mathrm{mV}$.

\section{Histological Procedures}

At the end of each experiment, animals were deeply anesthetized with sodium pentobarbital $(100 \mathrm{mg} / \mathrm{kg}$, intraperitoneally), and intracardially perfused first with heparinized saline and then with formalin. The brains were extracted, cryoprotected with a $30 \%$ sucrose solution, 
frozen, sectioned $(50 \mu \mathrm{m})$ with a cryostat, mounted on microscope slides and stained. Verification of microdialysis cannulae placements was performed using cresyl violet. The center of the injection site was readily identified by the Evans Blue dye, which remained visible for months after local administration of saline or TTX. Sections were stained using Nuclear Fast Red.

\section{Statistical Analyses}

The effects of NAC infusions (aCSF or NMDA, in all experiments) and amphetamine (in the PD7 VH group) were tested using a mixed design. Dialysis sessions were counterbalanced across within-subject drug and vehicle conditions, allowing each subject to act as its own control. Age and brain region sustaining inactivation were betweensubject factors. In each experiment, baseline (collection nos. 1-4) values of extracellular ACh (fmol/15 $\mu \mathrm{l})$ were compared using one-way repeated measures analysis of variance (ANOVA) for differences as a function of either dialysis session or treatment group. In the absence of significant differences, subsequent drug effects were then expressed as a percentage of the mean basal $\mathrm{ACh}$ value for that particular treatment group. For the initial experiment on agedependent effects of $\mathrm{VH}$ inactivation, an overall ANOVA was conducted on ACh values using a four-way ANOVA with AGE and TREATMENT GROUP as between-subject factors and DRUG and TIME as within-subject factors. Following a significant omnibus ANOVA, three- and twoway ANOVAs were conducted to identify the sources of main effects and interactions. For additional experiments (DH, NAC, or BLA inactivation), an initial three-way ANOVA was conducted, followed by a series of two-way ANOVAs. In all ANOVAs, the Huynh-Feldt correction was utilized to reduce Type I errors associated with repeated measures ANOVAs (Vasey and Thayer, 1987). When appropriate, a minimum number of post hoc comparisons were conducted using Bonferroni ( $\alpha$-adjusted) $t$-tests. All statistical tests were performed using SPSS for Windows (V15.0; SPSS, Chicago, IL).

\section{RESULTS}

\section{Infusions of Saline or TTX into VH}

Figure 1 shows photomicrographs of coronal sections depicting representative placements of TTX into the $\mathrm{VH}$, DH, BLA, and NAC. Injection sites were evidenced by small spots of dye, $10-30 \mu \mathrm{m}$ in diameter. There were no obvious differences between the characteristics of the gliotic spots produced by infusions into the VH at PD7 (Figure 1a) or PD32 (Figure 1b), or by PD7 infusions into the DH, amygdala, or NAC (Figure 1c-e). Only rats with placements consistent with these representatives were used in these studies $(n=5-7 /$ treatment/region). The following number of animals (collapsed between sham and TTX) were discarded owing to misplaced injections: $\mathrm{nVH}$ PD7 $=5$; $\mathrm{nVH} \mathrm{PD} 32=2 ; \mathrm{nDH} \mathrm{PD} 7=2 ; \mathrm{nBLA} \mathrm{PD} 7=4$; and $\mathrm{nNAC}$ $\mathrm{PD} 7=3$.

Rats were extensively habituated to the microdialysis testing bowls. Thus, they were largely at rest during the test period. Following the NMDA perfusions into the NAC shell, there was often an increase in the level of alertness and an orientation toward persons coming into the room to collect dialysis samples, but that was the extent of the change in behavior. There were no differences among the control vs TTX treatment groups in this regard. We did observe, in the few animals that mistakenly had dialysis probes into the NAC core (not used in this study), a distinct increase in motoric activity.

\section{Age-Dependent Effects of Ventral Hippocampal Inactivation}

The effects of NAC perfusion of NMDA on prefrontal ACh release in rats treated with intra- $\mathrm{VH}$ infusions of saline or TTX on PD7 or PD32 are illustrated in Figures 2 and 3, respectively. Mean ( \pm SEM) absolute basal ACh levels, collapsed across inactivation condition and drug treatment groups, at PD7 and PD32 were $7.01 \pm 0.29$ and $7.00 \pm$ $0.24 \mathrm{fmol} / 15 \mu \mathrm{l}$, respectively. Given that there were no significant differences between basal ACh efflux on the basis of AGE, INACTIVATION CONDITION, or DRUG
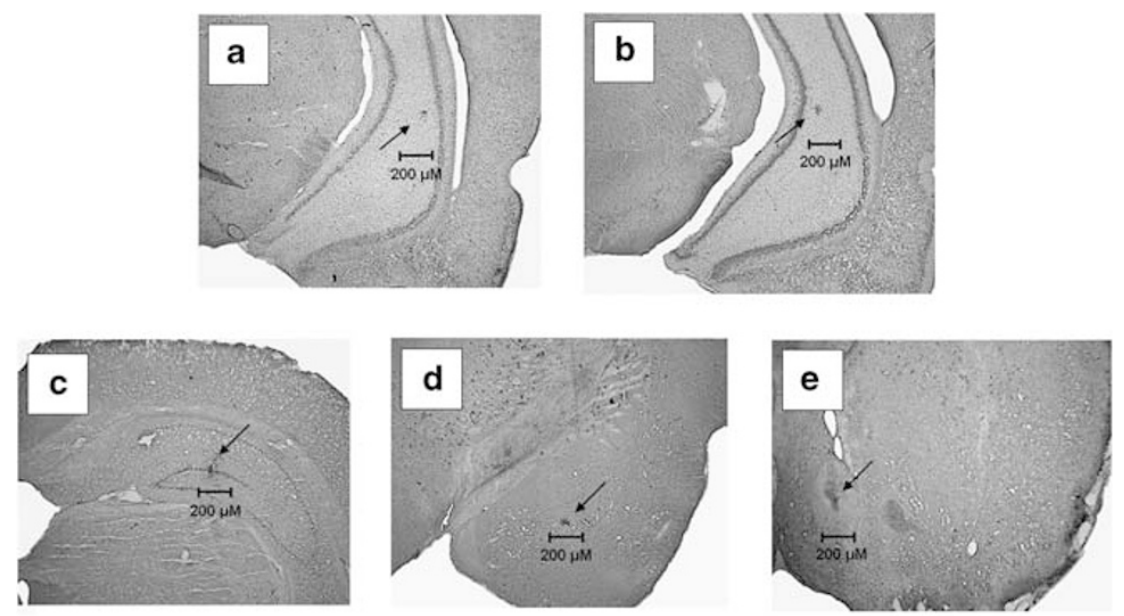

Figure I Nissl-stained coronal sections showing spots of dye indicating the placement of tetrodotoxin (TTX) injections into the ventral hippocampus at postnatal day (PD)7 (a) and PD32 (b), and the dorsal hippocampus (c), the amygdala (d), and the nucleus accumbens (e; all at PD7). 


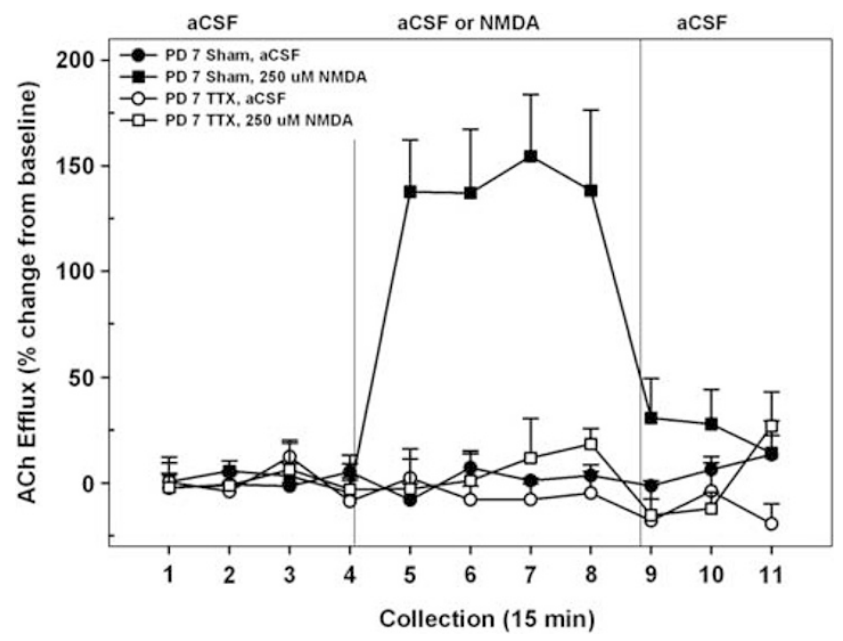

Figure 2 Mean $( \pm$ SEM) acetylcholine $(A C h)$ efflux in the medial prefrontal cortex (mPFC) of postnatal day (PD)7 nVHTTX rats $(n=8)$ and sham controls $(n=9)$ receiving, in pseudo-randomized order, perfusions of vehicle (artificial cerebral spinal fluid (aCSF)), or N-methyl-D-aspartate (NMDA) $(250 \mu \mathrm{M})$ into the nucleus accumbens (NAC) shell during separate dialysis sessions as adults. Following baseline (collection nos. 1-4), aCSF or NMDA was perfused for I h (collection nos. 5-8). Following drug perfusion, aCSF was re-perfused for 45 min (collection nos. 9-I I) until the conclusion of the dialysis session. Perfusion of NMDA resulted in a robust and sustained increase in cortical ACh efflux, above that observed during aCSF perfusion, in sham animals (collection nos. 5-8). In nVHTTX animals, however, perfusion of NMDA failed to increase cortical ACh efflux above baseline levels.

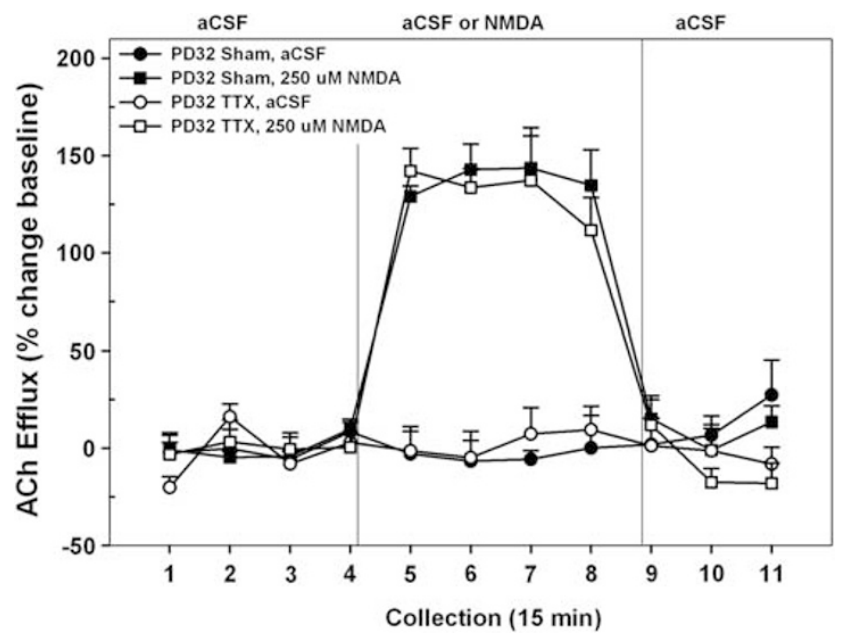

Figure 3 Mean $( \pm$ SEM) acetylcholine (ACh) efflux in the medial prefrontal cortex (mPFC) of postnatal day (PD)32 VHTTX rats $(n=8)$ and sham controls $(n=7)$ receiving, in pseudo-randomized order, perfusions of vehicle (artificial cerebral spinal fluid (aCSF)) or N-methyl-D-aspartate (NMDA) $(250 \mu \mathrm{M})$ into the nucleus accumbens (NAC) shell during separate dialysis sessions as adults. Following baseline (collection nos. I-4), aCSF or NMDA was perfused for I h (collection nos. 5-8). Following drug perfusion, aCSF was re-perfused for 45 min (collection nos. 9-1 I) until the conclusion of the dialysis session. Both control and VHTTX animals demonstrated a marked increase in cortical ACh efflux above that observed during aCSF perfusion (collection nos. 5-8). There was no effect of VHTTX inactivation on the NMDA-stimulated release.

TREATMENT (all F's $<3.52$ ), subsequent analyses were conducted on ACh levels expressed as a percent of mean basal efflux.
A comparison of Figures 2 and 3 reveals that the ability of NMDA receptors in NAC to stimulate prefrontal ACh release differed between controls and rats sustaining transient inactivation of the VH with TTX (INACTIVATION CONDITION, $\mathrm{F}_{1,28}=38.58, p<0.001$ ) and that the impact of the hippocampal inactivation on NMDA stimulation differed between the two ages (INACTIVATION CONDITION $\times$ AGE $\times$ DRUG $\times$ TIME $\left., \quad F_{10,28}=3.22, \quad p=0.001\right)$. More selective ANOVAs, restricted to a single age, revealed the sources of this interaction. When animals received VH infusions on PD7 and were subsequently tested as adults (Figure 2), control rats exhibited a robust increase in prefrontal ACh following NMDA, whereas TTX-treated rats did not (INACTIVATION CONDITION $\times$ DRUG $\times$ TIME, $\left.\mathrm{F}_{20,300}=4.57, p<0.001\right)$. In controls, cortical ACh levels rose $(\sim 130 \%$ above basal and aCSF-treated control conditions) within $15 \mathrm{~min}$ after the first NMDA perfusion interval (interval no. 5; $p<0.001$ ) and returned to basal values upon termination of the drug perfusion (interval no. 9).

When animals received VH infusions on PD32 and were subsequently tested as adults (Figure 3 ), there was a close similarity in the ability of NAC-NMDA perfusions to stimulate cortical ACh release in saline- and TTX-treated rats (INACTIVATION CONDITION $\times$ DRUG $\times$ TIME, $\mathrm{F}<0.39$ ). In both groups, the infusion of NMDA resulted in a $125-150 \%$ increase in prefrontal ACh levels relative to both baseline and those seen during the aCSF control condition. Again, this effect was evident at the end of the first collection interval (no. $5, p<0.001$ ) and back to normal within 15 min of drug termination (no. 9).

Two additional drug conditions were assessed in these adult rats treated with intra-hippocampal saline or TTX on PD7 to further characterize the insensitivity to NMDA in the inactivated group. To determine whether the apparent insensitivity of TTX-treated rats reflected a subsensitivity of the NMDA receptor, we tested rats with an intra-NAC perfusion of a higher dose of NMDA $(400 \mu \mathrm{M})$. Again, control rats receiving intra- $\mathrm{VH}$ saline on PD7 responded to the perfusion of NMDA with a marked elevation in prefrontal ACh (maximum mean $( \pm$ SEM) increase above baseline $=146 \pm 19 \%$ ), whereas TTX-treated rats continued to be insensitive to the NMDA perfusion (maximum increase $=12 \pm 18 \%$; INACTIVATION CONDITION, $\mathrm{F}_{1,15}=39.51, p<0.001 ;$ INACTIVATION CONDITION $\times$ DRUG $\times$ TIME, $\mathrm{F}_{10,150}=8.30, p<0.001$; data not shown).

In addition, on the final dialysis session all P7-treated rats received a systemic injection of amphetamine $(2.0 \mathrm{mg} / \mathrm{kg}$, i.p.) to determine whether the BFCS remained responsive to other pharmacological stimulations. We and others have repeatedly shown that systemic amphetamine increases cortical ACh release in intact rats (Day et al, 1994; Nelson et al, 2000; Arnold et al, 2001) and, importantly, this stimulation does not involve amphetamine's actions within the NAC (Arnold et al, 2000). Thus, amphetamine was used as a positive control for activity within the BFCS (Alexander et al, 2009). Amphetamine markedly stimulated prefrontal ACh levels in both control and inactivated rats (Figure 4; DRUG, $\left.F_{1,9}=263.17, p<0.001\right)$. Moreover, there were no significant differences between the magnitude and time course of the stimulation in saline- and TTX-treated rats $($ INACTIVATION CONDITION $\times$ DRUG $\times$ TIME, $\mathrm{F}<0.69$ ) 


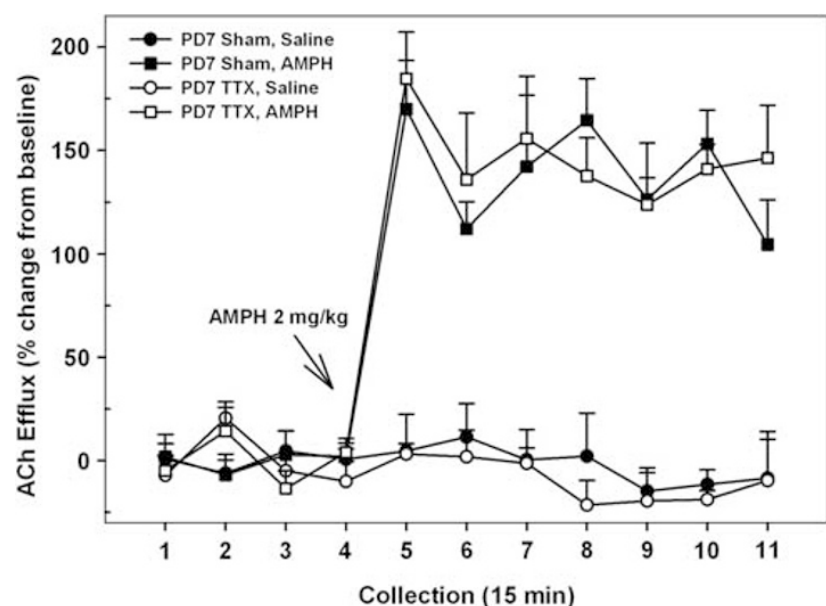

Figure 4 Mean $( \pm S E M)$ acetylcholine (ACh) efflux in the medial prefrontal cortex (mPFC) of postnatal day (PD)7 VHTTX rats $(n=6)$ and sham controls $(n=5)$ receiving systemic injections of amphetamine (AMPH; $2 \mathrm{mg} / \mathrm{kg}$, intraperitoneally) or saline as adults. Following baseline (collection nos. I-4), AMPH or saline was injected. Administration of $\mathrm{AMPH}$ resulted in significant and equivalent increases in cortical $\mathrm{ACh}$ efflux in both sham and nVHTTX animals that began in collection no. 5 and persisted for the duration of the dialysis session (collection no. I I).

In both groups, ACh levels are elevated during the first collection interval (no. 5) after the injection and remained so for the duration of the session (all $p$ 's $<0.001$ ).

\section{Effects of Transient Inactivation of Other Forebrain Regions}

TTX infusions into the DH. The effects of transient TTXinduced inactivation of the DH on PD7 on subsequent NMDA-mediated prefrontal ACh levels are summarized in Figure 5. The mean basal cortical ACh release in all rats receiving intra-DH infusions was $4.97 \pm 0.10 \mathrm{fmol} / 15 \mu \mathrm{l}$. As baseline levels did not differ as a function of INACTIVATION CONDITION or DRUG TREATMENT (both F's $<4.64$ ), subsequent analyses were conducted on ACh levels expressed as changes from these basal values. In marked contrast to the effects in rats treated on PD7 with intra-VH infusions (Figure 2), TTX administration into the DH on PD7 had no effect on the subsequent ability of intraNAC infusions of NMDA to stimulate prefrontal ACh release (Figure 5). Overall, NMDA infusions produced a robust increase in prefrontal ACh levels (DRUG TREATMENT, $\left.F_{1,9}=113.21 ; p<0.0001\right)$. However, there were no differences in the magnitude or time course of this stimulation in sham- or TTX-treated rats (INACTIVATION CONDITION; DRUG $\times$ INACTIVATION CONDITION $\times$ TIME, both F's $<0.76$ ). In saline- and TTX-treated rats, the NMDA-stimulated ACh levels, relative to aCSF controls, was evident by collection no. 5 (both $p$ 's $<0.01$ ) and returned to basal values by collection no. 9 (both p's $>0.5$ ).

TTX infusions into the BLA. The effects of transient TTXinduced inactivation of the BLA on PD7 on subsequent NMDA-mediated prefrontal ACh levels are summarized in Figure 6. The mean basal cortical ACh release in all rats

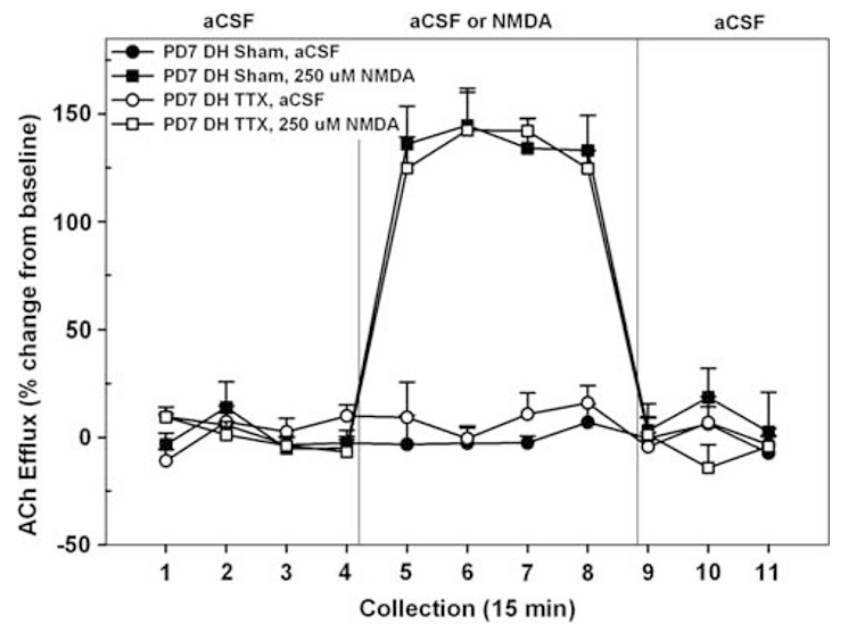

Figure 5 Mean $( \pm S E M)$ acetylcholine (ACh) efflux in the medial prefrontal cortex (mPFC) of postnatal day (PD)7 nDHTTX rats $(n=6)$ and sham controls $(n=5)$ receiving, in pseudo-randomized order, perfusions of vehicle (artificial cerebral spinal fluid aCSF) or N-methyl-D-aspartate (NMDA) $(250 \mu \mathrm{M})$ into the dorsal hippocampus $(\mathrm{DH})$ during separate dialysis sessions as adults. The period for baseline infusions of aCSF and administration of NMDA was the same as those reported in previous experiments (Figures 2 and 3). In contrast to the effects seen following inactivation of the ventral hippocampus $(\mathrm{VH})$ (Figure 2), administration of NMDA resulted in robust increases in cortical ACh efflux in both sham and nDHTTX groups (collection nos. 5-8).

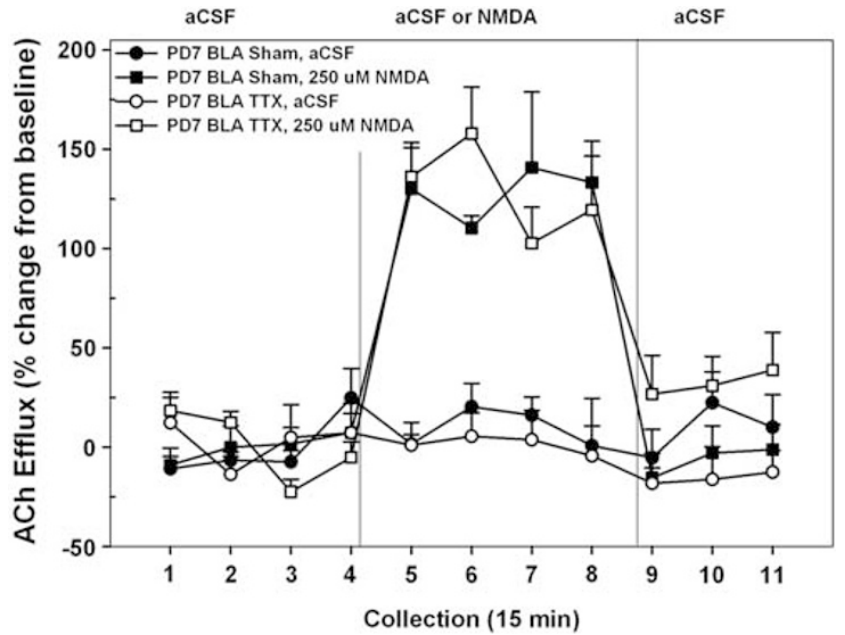

Figure 6 Mean $( \pm S E M)$ acetylcholine $(A C h)$ efflux in the medial prefrontal cortex (mPFC) of postnatal day (PD)7 nBLATTX rats $(n=6)$ and sham controls $(n=5)$ receiving, in pseudo-randomized order, perfusions of vehicle (artificial cerebral spinal fluid (aCSF)) or N-methyl-D-aspartate (NMDA) $(250 \mu \mathrm{M})$ into the nucleus accumbens (NAC) shell during separate dialysis sessions as adults. The period for baseline infusions of aCSF and administration of NMDA was the same as those reported in previous experiments (Figures 2 and 3). In contrast to the effects seen in the nVHTTX inactivation group (Figure 2), administration of NMDA resulted in robust increases in cortical ACh efflux in both sham and nBLATTX groups (collection nos. 5-8), indicating that inactivation of the basolateral amygdala (BLA), at this time in development, does not disrupt NMDA-induced cortical $A C h$ release.

receiving intra-BLA infusions was $7.05 \pm 0.82 \mathrm{fmol} / 15 \mu \mathrm{l}$. As baseline levels did not differ as a function of INACTIVATION CONDITION or DRUG TREATMENT (both F's $<0.06$ ), subsequent analyses were conducted on 
ACh levels expressed as changes from these basal values. Unlike the case of rats treated on PD7 with intra-VH infusions, TTX administration into the BLA had no effect on the subsequent ability of NAC NMDA receptors to stimulate prefrontal ACh release. Overall, drug perfusion resulted in a marked elevation of ACh levels (DRUG TREATMENT, $\left.\mathrm{F}_{1,9}=69.42 ; p<0.001\right)$. Moreover, there was no significant difference between rats infused with saline or TTX, into the BLA, on the NMDA-based modulation of cortical ACh levels (INACTIVATION CONDITION; DRUG $\times$ INACTIVATION CONDITION $\times$ TIME, both F's $<1.71)$. In saline- and TTXtreated rats, NMDA markedly stimulated ACh levels, relative to aCSF controls, and this effect was evident by collection no. 5 (both $p$ 's $<0.01$ ) and returned to basal values by collection no. 9 (both $p$ 's $>0.5$ ). This profile of NMDA-modulated ACh release in PFC closely resembled that seen in rats initially infused with saline into the $\mathrm{VH}$ on PD7 (Figure 2) or rats infused with saline or TTX into the $\mathrm{DH}$ on PD7 (Figure 5) or into the $\mathrm{VH}$ on PD32 (Figure 3).

TTX infusions into the NAC. The effects of transient TTXinduced inactivation of the NAC on PD7 on subsequent NMDA-mediated prefrontal ACh levels are summarized in Figure 7. The mean basal cortical ACh release in all rats receiving intra-NAC infusions was $5.10 \pm 0.14 \mathrm{fmol} / 15 \mu \mathrm{l}$. As baseline levels did not differ as a function of INACTIVATION CONDITION or DRUG TREATMENT (both F's $<3.23$ ), subsequent analyses were conducted on $\mathrm{ACh}$ levels expressed as changes from these basal values. Unlike the case of rats treated with intra-hippocampal TTX on PD7 and as reported above in the case of $\mathrm{DH}$ or BLA

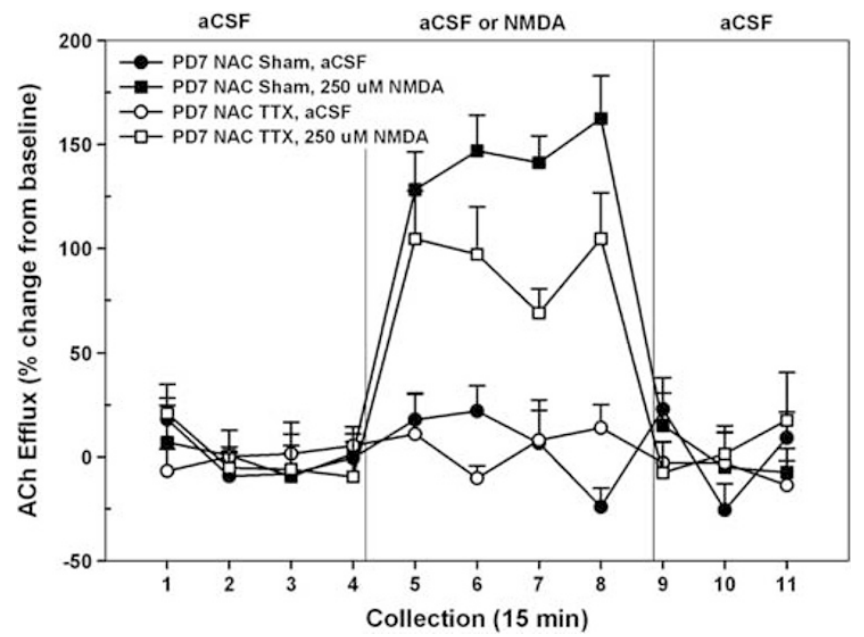

Figure 7 Mean $( \pm S E M)$ acetylcholine (ACh) efflux in the medial prefrontal cortex (mPFC) of postnatal day (PD)7 nNACTTX rats $(n=5)$ and sham controls $(n=6)$ receiving, in pseudo-randomized order, perfusions of vehicle (artificial cerebral spinal fluid $(\mathrm{aCSF})$ ) or $\mathrm{N}$-methyl-D-aspartate (NMDA) $(250 \mu \mathrm{M})$ into the nucleus accumbens (NAC) shell during separate dialysis sessions as adults. The period for baseline infusions of aCSF and administration of NMDA was the same as those reported in previous experiments (Figures 2 and 3 ). In contrast to the findings in Figure 2, perfusion of NMDA resulted in an increase in cortical ACh efflux, above that observed during aCSF perfusion, in both sham and nNACTTX animals (collection nos. 5-8). However, the responsivity of nNACTTX rats to NMDA was attenuated and only sham-lesioned rats exhibited a druginduced stimulation of ACh that was similar to those previously reported. inactivation, TTX administration into the developing NAC did not eliminate the subsequent ability of NAC NMDA receptors to stimulate prefrontal ACh release. Overall, NMDA perfusion resulted in significant elevations of cortical ACh levels (DRUG TREATMENT, $\mathrm{F}_{1,10}=6.23$, $p<0.001$ ), but there was no effect of TTX treatment (INACTIVATION CONDITION, $\mathrm{F}<1.89$ ). The data in Figure 7 suggest time-related treatment differences in the magnitude of the NMDA response between the two groups and this was supported statistically (DRUG TREATMENT $\times$ INACTIVATION CONDITION $\times$ TIME, $\left.\mathrm{F}_{10,100}=2.29, p=0.02\right)$. A two-way ANOVA comparing the effect of NMDA perfusion over time between the shamand TTX-treated groups revealed that neonatal inactivation of the NAC on PD7 led to a subsequent reduction in the magnitude of stimulated release when animals were tested as adults (DRUG TREATMENT $\times$ TIME, $\quad \mathrm{F}_{10,50}=1.94$, $p=0.05)$.

\section{DISCUSSION}

These experiments extend our previous report of impaired mesolimbic regulation of the prefrontal cortical cholinergic system following permanent excitotoxic lesions of the developing VH (Alexander et al, 2009) by demonstrating a similar dysregulation following a more subtle insult; transient interruption of $\mathrm{Na}^{+}$-gated impulse flow in $\mathrm{VH}$ with TTX. Infusions of TTX into VH on PD7 eliminated the well-established ability of intra-accumbens NMDA perfusions to evoke prefrontal $\mathrm{ACh}$ release in intact adult rats (Brooks et al, 2007; Zmarowski et al, 2005). This elimination of NMDA-stimulated release did not reflect a generalized reduction in the capacity of the corticopetal cholinergic system to respond to stimuli as a control experiment demonstrated that cortical ACh levels were elevated following systemic amphetamine, a pharmacological stimulus previously shown not to involve the NAC (Arnold et al, 2000). Importantly, the disruptive effects of TTX infusions were age-specific in that similar VH infusions on PD32 had no effect on the subsequent NMDA-evoked ACh release in PFC. Moreover, the effects were also regionally selective as TTX infusions into DH, BLA, or the shell region of the NAC on PD7 did not disrupt the maturation of this effect (although the NAC inactivation resulted in an attenuated response to NMDA; see below). In the sections that follow, we discuss: (a) potential mechanisms associated with ageand regional-dependent effects of TTX on the maturation of the mesolimbic regulation of the corticopetal cholinergic system and (b) the potential validity of transient inactivation of ventral hippocampal outflow as an animal model for studying the cognitive deficits seen in SZ.

\section{Age- and Regional-Dependent Effects of TTX Infusions}

Several studies have addressed the extent of spread following intracranial administration of TTX and the duration of its effects on neuronal excitability in vivo. Using an intracerebral injection of TTX $(31.3 \mu \mathrm{M})$ into the pre-ganglionic parasympathetic nucleus controlling the muscles of the iris, Zhuravin and Bures (1991) demonstrated that TTX rapidly blocked transmission, resulting in 
pupil dilation that reached maximum effect at $40 \mathrm{~min}$, remained at that level for $2 \mathrm{~h}$, and then slowly decayed over the next $20 \mathrm{~h}$. Boehnke and Rasmusson (2001) perfused TTX $(10 \mu \mathrm{M})$ into the raccoon somatosensory cortex and then measured evoked neural responses at various distances from the dialysis probe. They reported significant decreases in responding as far as $2.0 \mathrm{~mm}$ away from the delivery site that persisted, at maximal levels, for over $2 \mathrm{~h}$ (no data are provided for latency to return to baseline). van Duuren et al (2007) perfused TTX $(1.0 \mu \mathrm{M})$ into orbital and lateral regions of PFC and used a multi-tetrode array to determine neuronal firing rates $0.48-0.62 \mathrm{~mm}$ away. Reductions in neuronal activity were rapid, yet still partially evident after $2.5 \mathrm{~h}$. Finally, the effects of TTX perfusion within the striatum were measured in terms of reductions in recovered basal DA levels from a dialysis probe located $1 \mathrm{~mm}$ from the perfusion site. Within $10 \mathrm{~min}$, DA levels had fallen by $80 \%$, but had recovered to a $40 \%$ decline 10 min later (Westerink and De Vries, 2001). On the basis of these studies, it seems clear that our localized infusions of TTX had their most enduring effects, lasting several hours, close to the injection site, but also exhibited significant spread beyond the intended target region.

Intra-VH infusions of TTX on PD7 markedly disrupted the subsequent mesolimbic control of cortical cholinergic transmission, yet similar infusions on PD32 were without effect. These data suggest that neuronal events critical to the functional maturation of this distributed system are ongoing during earlier, but not later ages of development, and that these mechanisms contribute to subsequent deficits in the modulation of cortical cholinergic transmission exhibited in PD7 rats. This impaired regulation of cortical ACh levels may reflect local changes in cortical mechanisms (Nair and Gudelsky, 2004; Parkih et al, 2008) and/or longloop, trans-synaptic changes affecting the excitability of the BFCS projections. Several mechanisms could contribute to these developmental effects. First, the TTX infusions on PD7 may produce functional deficits in developing GABAergic interneurons critical for regulating the excitability of $\mathrm{VH}$ projections (Francois et al, 2009; Lipska et al, 2003) to target neurons in PFC through either direct projections (Jay et al, 1989; Tierney et al, 2004; White et al, 1990) or indirectly via BLA (Sarter and Markowitsch, 1984) or NAC by way of basal forebrain (Henny and Jones, 2008) or medial dorsal thalamus (Sarter and Markowitsch, 1984). Second, TTXinduced blockade of neurotransmission may bias the activity-dependent competition of axons for synaptic space in hippocampus and/or its cortical or limbic target regions (Hua et al, 2005; Katz and Shatz, 1996). Evidence suggests that these various inputs are arriving and establishing synaptic contacts in rat cortex by PD10 and continuing through PD26-30. The formation of mature spines in cultured granule cells is reduced following exposure to TTX and the resultant immature spines are less likely to make functional synaptic contacts (Drakew et al, 1999). In this regard, reductions in cortical and hippocampal spine density have been reported in the brains of SZ patients (Lewis and Gonzalez-Burgos, 2008). Third, TTX-induced reductions in functional activity may result in populations of affected neurons being more susceptible to anomalous pruning during the adolescent-puberty period (Teicher et al, 2003). The well-known manifestation of a SZ-like phenotype at the time of late puberty in these various hippocampal-based models (Alexander et al, 2009; Tseng et al, 2007) of the disorder may relate, in part, to the timing of this inappropriate pruning. Finally, TTX-induced changes in impulse flow may diminish myelination of hippocampal and other projection neurons, during the sensitive period around PD7, but not PD32 (Suzuki and Raisman, 1994; Zalc and Fields, 2000). It is possible that changes in the myelination of hippocampal projection neurons during periods of innervations in PFC may dysregulate cortical ACh release, uncouple the synchrony of neuronal oscillations, and, as a result, produce the cognitive deficits in seen SZ (Ford et al, 2007; Jones, 2010).

NMDA perfusions in rats treated with intra-NAC TTX on PD7 exhibited a significant increase in cortical ACh levels. However, the magnitude of this release was attenuated relative to that seen in sham-treated controls. The mechanisms contributing to this effect are unknown, but may include a reduction in the number or sensitivity of NMDA receptors on the GABAergic projection neurons to basal forebrain. The time frame between PD4 and PD14 represents a stage where EPSCs measured in NAC slices are predominantly driven by NMDA receptor activation (Zhang and Warren, 2008). These authors speculated that this represents a developmental period during which the maturation of the NAC might be most sensitive to environmental manipulation such as, in the case of these experiments, a reduction of excitatory input from the $\mathrm{VH}$.

\section{The Validity of the VH TTX Inactivation Model for SZ}

The anterior aspect of the hippocampal formation is one of the brain regions most consistently altered in SZ (Goldman and Mitchell, 2004; Harrison, 2004; Tamminga et al, 2010). Moreover, many of the cognitive deficits seen in the disorder are mediated by dysregulated interactions between the hippocampus and its principal target regions such as PFC (Henseler et al, 2010; Wolf et al, 2009), NAC (Chambers et al, 2001; Csernansky et al, 1993), and ventral tegmental area (Yang et al, 1999; Lisman et al, 2010). Thus, manipulations that alter the maturation of the VH (rodent counterpart of anterior hippocampal formation; Dolorfo and Amaral, 1998) represent rational animal models for studying the cognitive deficits seen in SZ. The best studied of these models, ibotenic acid-induced lesions of VH during early development, enjoys a significant amount of face and predictive validity (for a review see, Lipska and Weinberger, 2002c; Tseng et al, 2009). However, the resulting massive loss of $\mathrm{VH}$ tissue far exceeds the more subtle cellular disorientations, reductions in synaptic density, and changes in protein expression reported in the brains of SZ patients (Harrison, 2004; Tamminga et al, 2010).

Transient inactivation of the $\mathrm{VH}$ during this early sensitive period may ultimately represent an animal model that possesses a greater degree of construct validity than the permanent lesion model. Although the maturational impact of this selective interruption of neuronal transmission, on neurobiological or cognitive end points, has not yet received the considerable attention devoted to other animal models, the few studies that have been conducted indicate effects that are consistent with the SZ phenotype. SZ patients exhibit a characteristic impairment in performance on 
latent inhibition tasks (Gray, 1998; Gray and Snowden, 2005). Rats receiving TTX infusions into entorhinal cortex or ventral subiculum as neonates, but not as adults, exhibit deficits in latent inhibition (Meyer et al, 2009; Peterschmitt et al, 2007). Moreover, these deficits in latent inhibition are accompanied by a disruption of the normal coupling between performance in the task and striatal dopamine release (Meyer et al, 2009).

We have used activation of NMDA receptors in the NAC shell as a pharmacological mimic of the top-down activation of prefrontal glutamatergic inputs to this motivation-toattention circuit. In intact animals, perfusion of NMDA into the NAC shell markedly stimulates basal ACh levels in PFC (Zmarowski et al, 2005), and this NMDA modulation is facilitated by concurrent activation of D1 receptors in NAC (Zmarowski et al, 2005) and inhibited by stimulation of D2 receptors (Brooks et al, 2007). Recently, we demonstrated that infusions of NMDA into NAC shell rescued the falling performance of rats performing in a sustained attention task when faced with distractors that otherwise severely impair attentional processing (St Peters et al, 2011). Thus, NMDA-mediated elevation of prefrontal ACh levels serves as a bioassay for testing the integrity and efficacy of topdown control mechanisms that are essential for maintaining goal-directed behavior.

As a cognitive parallel to the impaired mesolimbic modulation of cortical cholinergic transmission reported in this manuscript, we also recently reported that rats sustaining TTX infusions of the VH on PD7 exhibit reversal and extra-dimensional shift deficits in an attentional setshifting task. Importantly, impairments were not seen in rats receiving TTX into DH on PD7 or into VH on PD32; thus, these emerging cognitive data closely parallel the neurochemical data reported in this article (Brooks et al, 2010, unpublished observations). Finally, using a sustained attention task, we recently found that as adults, animals that received nVHTTX infusions on PD7, but not PD32, exhibit an impairment in the consolidation of changes in attentional performance triggered by changing the probability of signal vs non-signal trials and the reward for correct responses (H Gritton et al, unpublished). Collectively, these findings are consistent with deficient cognitive flexibility in PD7 animals and highlight the heuristic leverage provided by this animal model for the study of neuronal mechanisms underlying, and associated treatment strategies for, the cognitive symptoms of SZ.

\section{ACKNOWLEDGEMENTS}

The authors' research was supported by a research grant from the National Institute of Health (MH057436) to JPB and MS.

\section{DISCLOSURE}

Listing for each author, detailing the names of organizations, institutions, companies, and individuals, including intermediaries such as sub-contractors or conference organizers, from whom they have received compensation for professional services in any of the previous 3 years, or from whom they anticipate receiving such compensation in the near future, whether or not these affiliations appear to have any relevance to the topic covered in the submission: John P Bruno: Nothing to report. Martin Sarter: Dr Sarter has received honoraria for speaking at Abbott Laboratories, Pfizer Pharmaceuticals, Lilly UK, and has received research support from Abbott Laboratories and Pfizer Pharmaceuticals. Julie M Brooks: Dr Brooks has nothing to disclose.

\section{REFERENCES}

Alexander KS, Brooks JM, Sarter M, Bruno JP (2009). Disruption of mesolimbic regulation of prefrontal cholinergic transmission in an animal model of schizophrenia and normalization by chronic clozapine treatment. Neuropsychopharmacology 34: 2710-2720.

Arnold HM, Fadel J, Sarter M, Bruno JP (2001). Amphetaminestimulated cortical acetylcholine release: role of the basal forebrain. Brain Res 894: 74-87.

Arnold HM, Nelson CL, Neigh GN, Sarter M, Bruno JP (2000). Systemic and intra-NAC accumbens administration of amphetamine differentially affects cortical acetylcholine release. Neuroscience 96: 675-685.

Boehnke SE, Rasmusson DD (2001). Time course and effective spread of lidocaine and tetrodotoxin delivered via microdialysis: an electrophysiological study in cerebral cortex. J Neurosci Methods 105: 133-141.

Brady AM, Saul RD, Wiest MK (2010). Selective deficits in spatial working memory in the neonatal ventral hippocampal lesion rat model of schizophrenia. Neuropharmacology 59: 605-611.

Brooks JM, Sarter M, Bruno JP (2007). D2-like receptors in nucleus accumbens negatively modulate acetylcholine release in prefrontal cortex. Neuropharmacology 53: 455-463.

Chambers RA, Krystal JH, Self DW (2001). A neurobiological basis for substance abuse comorbidity in schizophrenia. Biol Psychiatry 50: 71-83.

Csernansky JG, Wrona CT, Bardgett ME (1993). Subcortical dopamine and serotonin turnover during acute and subchronic administration of typical and atypical neuroleptics. Psychopharmacology 110: 145-151.

Day JC, Tham CS, Fibiger HC (1994). Dopamine depletion attenuates amphetamine-induced increases of cortical acetylcholine release. Eur J Pharmacol 263: 285-292.

Dolorfo CL, Amaral DG (1998). Entorhinal cortex of the rat: organization of intrinsic connections. J Comp Neurol 398: 49-82.

Drakew A, Frotscher M, Heimrich B (1999). Blockade of neuronal activity alters spine maturation of dentate granule cells but not their dendritic arborization. Neuroscience 94: 767-774.

Flores G, Barbeau D, Quirion R, Srivastava LK (1996). Decreased binding of dopamine D3 receptors in limbic subregions after neonatal bilateral lesion of rat hippocampus. J Neurosci 16: 2020-2026.

Francois J, Ferrandon A, Koning E, Angst M-J, Sandner G, Nehling A (2009). Selective reorganization of GABAergic transmission in neonatal ventral hippocampal-lesioned rats. Int J Neuropsychopharmacol 12: 1097-1110.

Ford JM, Krystal JH, Mathalon DH (2007). Neural synchrony in schizophrenia: from networks to new treatments. Schizophr Bull 33: $848-852$.

Goldman MB, Mitchell CP (2004). What is the functional significance of hippocampal pathology in schizophrenia? Schizophr Bull 30: 367-392.

Gray JA (1998). Integrating schizophrenia. Schizophr Bull 24: 249-266.

Gray NS, Snowden RJ (2005). The relevance of irrelevance to schizophrenia. Neurosci Biobehav Rev 29: 989-999.

Guillin O, Abi-Dargham A, Laruelle M (2007). Neurobiology of dopamine in schizophrenia. Int Rev Neurobiol 78: 1-39. 
Harrison PJ (2004). The hippocampus in schizophrenia: a review of the neuropathological evidence and its pathophysiological implications. Psychopharmacology 174: 151-162.

Henny P, Jones BE (2008). Projections from the basal forebrain to prefrontal cortex comprise cholinergic, GABAergic and glutamatergic inputs to pyramidal cells or interneurons. Eur J Neurosci 27: 654-670.

Henseler I, Falkai P, Gruber O (2010). Disturbed functional connectivity within brain networks subserving domain-specific subcomponents of working memory in schizophrenia: relation to performance and clinical symptoms. J Psychiatric Res 44: 364-372.

Hua JK, Smear MC, Baler H, Smith SJ (2005). Regulation of axon growth in vivo by activity-based competition. Nature 434: 1022-1026.

Hyde TM, Crook JM (2001). Cholinergic systems and schizophrenia: primary pathology or epiphenomena? J Chem Neuroanat 22: 53-63.

Jay TM, Glowinski J, Thierry AM (1989). Selectivity of the hippocampal projection to the prelimbic area of the prefrontal cortex in the rat. Brain Res 505: 337-340.

Jones MW (2010). Errant ensembles: dysfunctional neuronal network dynamics in schizophrenia. Biochem Soc Trans 38: 516-521.

Katz LC, Shatz CJ (1996). Synaptic activity and the construction of cortical circuits. Science 274: 1133-1138.

Laplante F, Srivastava LK, Quirion R (2004). Alterations in dopaminergic modulation of prefrontal cortical acetylcholine release in post-pubertal rats with neonatal ventral hippocampal lesions. J Neuroch 89: 314-323.

Lewis DA, Gonzalez-Burgos G (2008). Neuroplasticity of neocortical circuits in schizophrenia. Neuropsychopharmacology 33: 141-165.

Lipska BK, Aultman JM, Verma A, Weinberger DR, Moghaddam B (2002a). Neonatal damage of the ventral hippocampus impairs working memory in the rat. Neuropsychopharmacology 27: 47-54.

Lipska BK, Halim ND, Segal PN, Weinberger DR (2002b). Effects of reversible inactivation of the neonatal ventral hippocampus on behavior in the adult rat. J Neurosci 22: 2835-2842.

Lipska BK, Lerman DN, Khaing ZZ, Weinberger DR (2003). The neonatal ventral hippocampal lesion model of schizophrenia: effects on dopamine and GABA mRNA markers in the rat midbrain. Eur J Neurosci 18: 3097-3104.

Lipska BK, Weinberger DR (2002c). A neurodevelopmental model of schizophrenia: neonatal disconnection of the hippocampus. Neurotox Res 4: 469-475.

Lisman JE, Coyle JT, Green RW, Javitt DC, Benes FM, Heckers S et al (2008). Circuit-based framework for understanding neurotransmitter and risk gene interactions in schizophrenia. Trends Neurosci 31: 234-242.

Lisman JE, Pi HJ, Zhang YC, Otmakhova NA (2010). A thalamohippocampal-ventral tegmental area loop may produce the positive feedback that underlies the psychotic break in schizophrenia. Biol Psychiatry 68: 17-24.

Marquis J-P, Goulet S, Dore FY (2008). Neonatal ventral hippocampus lesions disrupt extra-dimensional shift and alter dendritic spine density in the medial prefrontal cortex of juvenile rats. Neurobiol Learn Mem 90: 339-346.

Meyer F, Peterschmitt Y, Louilot A (2009). Postnatal functional inactivation of the entorhinal cortex or ventral subiculum has different consequences for latent inhibition-related striatal dopaminergic responses in adult rats. Eur J Neurosci 29: 2035-2048.

Mitchell CP, Grayson DR, Goldman MB (2005). Neonatal lesions of the ventral hippocampal formation alter GABA-A receptor subunit mRNA expression in adult rat frontal pole. Biol Psychiatry 57: 49-55.
Mouri A, Noda Y, Enomoto T, Nabeshima T (2007). Phencyclidine animal models of schizophrenia: approaches from abnormality of glutamatergic neurotransmission and neurodevelopment. Neurochem Int 51: 173-184.

Nair SG, Gudelsky GA (2004). Activation of $5-\mathrm{HT}_{2}$ enhances the release of acetylcholine in the prefrontal cortex and hippocampus of the rat. Synapse 15: 202-207.

Narahashi T (1972). Mechanism of action of tetrodotoxin and saitoxin on excitable membranes. Fed Proc 31: 1124-1132.

Nelson CL, Sarter M, Bruno JP (2000). Repeated pretreatment with amphetamine sensitizes increases in cortical acetylcholine release. Psychopharmacology 151: 406-415.

Nuechterlein KH, Luck SJ, Lustig C, Sarter M (2009). CNTRICS final task selection: control of attention. Schizophr Bull 35: 182-196.

Parkih V, Man K, Decker MW, Sarter M (2008). Glutamatergic contributions to nicotinic acetylcholine-receptor agonist-evoked cholinergic transients in the prefrontal cortex. J Neuroscience 28: 3769-3780.

Patterson PH (2009). Immune involvement in schizophrenia and autism: etiology, pathology, and animal models. Behav Brain Res 204: 313-321.

Peterschmitt Y, Meyer F, Louilot A (2007). Neonatal functional blockade of the entorhinal cortex results in disruption of accumbal dopaminergic responses observed in latent inhibition paradigm in adult rats. Eur J Neurosci 25: 2504-2513.

Sarter M, Markowitsch HJ (1984). Collateral innervation of the medial and lateral prefrontal cortex by amygdaloid, thalamic, and brain-stem neurons. J Comp Neurol 224: 445-460.

St Peters M, Demeter E, Lustig C, Bruno JP, Sarter M (2011). Enhanced control of attention by stimulating mesolimbiccorticopetal cholinergic circuitry. J Neurosci 31: 9760-9771.

Suzuki M, Raisman G (1994). Multifocal pattern of postnataldevelopment of the macroglial framework of the rat fimbria. Glia 12: 294-308.

Tamminga CA, Stan AD, Wagner AD (2010). The hippocampal formation in schizophrenia. Am J Psychiatry 167: 1178-1193.

Teicher MH, Krenzel E, Thompson AP, Andersen SL (2003). Dopamine receptor pruning during the peripubertal period is not attenuated by NMDA receptor antagonism in rat. Neurosci Lett 339: 169-171.

Tierney PL, Degentetais E, Thierry AM, Glowinski J, Gioanni Y (2004). Influence of the hippocampus on interneurons of the rat prefrontal cortex. Eur J Neurosci 20: 514-524.

Tseng KY, Chambers RA, Lipska BK (2009). The neonatal ventral hippocampal lesion as a heuristic neurodevelopmental model of schizophrenia. Behav Brain Res 204: 295-305.

Tseng KY, Lewis BL, Lipska BK, O’Donnell P (2007). Post-pubertal disruption of medial prefrontal cortical dopamine-glutamate interactions in a developmental animal model of schizophrenia. Biol Psychiatry 62: 730-738.

van Duuren E, van der Plasse G, van der Blom R, Joosten RNJMA, Mulder AB, Pennartz CMA et al (2007). Pharmacological manipulation of neuronal ensemble activity by reverse microdialysis in freely moving rats: a comparative study of the effects of tetrodotoxin, lidocaine, and muscimol. J Pharmacol Exp Therap 323: 61-69.

Vasey MW, Thayer JF (1987). The continuing problem of false positives in repeated measures ANOVA in psychophysiology-a multivariate solution. Psychophysiology 24: 479-486.

Welsh RC, Chen AC, Taylor SF (2010). Low-frequency BOLD fluctuations demonstrate altered thalamocortical connectivity in schizophrenia. Schizophr Bull 36: 713-722.

Westerink BHC, De Vries JB (2001). A method to evaluate the diffusion rate of drugs from a microdialysis probe through brain tissue. J Neurosci Methods 109: 53-58.

White TD, Tan AM, Finch DM (1990). Functional reciprocal connections of the rat entorhinal cortex and subicular complex 
with the medial frontal cortex: an in vivo intracellular study. Brain Res 533: 95-106.

Wolf RC, Vasic N, Sambataro F, Hose A, Frasch K, Schmid M et al (2009). Temporally anticorrelated brain networks during working memory performance reveal aberrant prefrontal and hippocampal connectivity in patients with schizophrenia. Prog Neuro-Psychopharmacol Biol Psychiatry 33: 1464-1473.

Yang CR, Seamans JK, Gorelova N (1999). Developing a neuronal model for the pathophysiology of schizophrenia based on the nature of electrophysiological actions of dopamine in the prefrontal cortex. Neuropsychopharmacology 21: 161-194.

Zalc B, Fields RD (2000). Do action potentials regulate myelination? Neuroscientist 6: 5-13.
Zhang L, Warren RA (2008). Postnatal development of excitatory postsynaptic currents in nucleus accumbens medium spiny neurons. Neuroscience 154: 1440-1449.

Zhuravin IA, Bures J (1991). Extent of the tetrodotoxin induced blockade examined by papillary paralysis elicited by intracerebral injection of the drug. Exp Brain Res 83: 687-690.

Zmarowski A, Sarter M, Bruno JP (2005). NMDA and dopamine interactions in the nucleus accumbens modulate cortical acetylcholine release. Eur J Neurosci 22: 1731-1740.

Zmarowski A, Sarter M, Bruno JP (2007). Glutamate receptors in the nucleus accumbens mediate regionally selective increases in cortical acetylcholine release. Synapse 61: $115-123$. 